\title{
Thermodynamic Properties of Unburned Reactant Mixtures for Different Kind of Fuels
}

\author{
Hasan Kayhan KAYADELEN
}

Yıldız Teknik Üniversitesi, Gemi İnşaatı ve Denizcilik Fakültesi, Türkiye

hkayhan@yildiz.edu.tr, ORCID ID: orcid.org/0000-0003-4292-3770

\begin{abstract}
Accurate estimation of adiabatic flame temperature and combustion product species play a key role in estimating performance and emission characteristics of internal combustion engines. This can be succeeded only with exact knowledge of temperature and thermodynamic properties of the unburned mixture. In this paper, thermodynamic properties of various fuels and fuel-air mixtures are presented. Differences in gas phase and liquid phase properties are discussed. Effect of equivalence ratio and unburned mixture temperature on enthalpy, specific heat and entropy of unburned fuel-air mixtures as well as effect of phase change on adiabatic flame temperature are shown. In order to serve as a recourse tool for researchers of combustion and internal combustion engines, the required polynomial coefficients used in calculation of thermodynamic properties are tabulated in famous NASA format for various important chemical compounds which are commonly used to represent surrogates of diesel, gasoline, Jet-A and many other fuels.
\end{abstract}

Keywords: Combustion, Fuels, Unburned Mixture, Thermodynamic Properties, NASA Format.

\section{Farklı Yakıtlara Ait Yanmamış Dolgu Karışımlarının Termodinamik Özeliklerinin Belirlenmesi}

$\ddot{O} z$

İçten yanmalı motorlarda yanma ürünleri ve adyabatik alev sıcaklığının hassas bir şekilde tayin edilmesi, performans ve kirletici emisyonların doğru olarak hesaplanabilmesinde önemli bir rol oynamaktadır. Bu da ancak yanmamıs dolguya ait termodinamik özeliklerin tam olarak bilinmesiyle mümkündür. Bu çalışmada çeșitli yakıt ve yakıt-hava karışımlarının, ayrıca bunlarla birlikte bulunan diğer reaktanların varlığında termodinamik özeliklerin tayin edilebilmesi için hassas sonuç veren bir metot tanıtılmış, yakıtların sıvı veya gaz fazında bulunmaları halinde termodinamik özelikler ve adyabatik alev sıcaklığında meydana gelen değişimler incelenmiştir. Bahsedilen metot kullanılarak diesel, benzin, ve jet-A gibi yakıtları oluşturan ve sıkça kullanılan yakıt bileșenlerinin sıvı hallerindeki termodinamik özeliklerinin hesaplanmasinda kullanılacak katsayılar, yaygın olarak kullanılan NASA formatında hesaplanarak tablolaştırılmış, içten yanmalı motorlar üzerine çalışan araştırmacıların istifadesine sunulmuştur.

Anahtar Kelimeler: Yanma, Yakıtlar, Yanmamış Dolgu, Termodinamik Özelikler, NASA Polinomları. 


\section{Introduction}

Improving cycle parameters such as thermal efficiency and work produced by an engine or reducing exhaust gas emissions are the main objectives of many studies on energy systems [1-4]. In order to calculate these cycle parameters precisely, we need an accurate computation of the changes of state due to combustion. Fuel-air mixtures are usually composed of more than one thermodynamic phase. Accordingly, rather than modeling the fuel-air mixture as an ideal gas mixture, a more realistic approach is to regard each individual species in their actual phases [5]. This task can be performed in two different ways, one of which is to provide the properties of the substances in a tabular form at predetermined temperature intervals and the values needed are calculated by interpolation. This method requires, handling thousands of thermodynamic values, large memory storage and does not permit use beyond the temperature limits of the table. A second and more commonly used technique is representation of the properties of each species by polynomials that allow direct calculation of the thermodynamic properties at any temperature. The polynomials obtained from fitting specific heat, entropy and enthalpy with simultaneous least squaring of are known as the NASA thermodynamic polynomials because of their use in a variety of NASA computer programs [6]. The NASA polynomials are usually fitted in the temperature range 300 to $5000 \mathrm{~K}$ one is for 300 to $1000 \mathrm{~K}$ and the other is from 1000 to $5000 \mathrm{~K}$ range [7]. Because the scope of this work is unburned mixture properties, polynomial coefficients of low temperature range ( 300 to $1000 \mathrm{~K}$ ) is of concern.

Numerous researches of internal combustion engines and gas turbines [817] use these polynomial coefficients in performance calculations which calculate thermodynamic properties matching that of famous combustion software CHEMKIN [18] and of Gaseq [19]. In most cases, these coefficients for individual substances can only be found for gas phase but gas phase polynomial coefficients cannot represent thermodynamic properties at liquid phase. Accordingly, direct usage of gas phase coefficients for calculation of unburned mixture properties cause deviations in thermodynamic properties which deteriorate performance parameters such as net cycle work, thermal efficiency and energy efficiency, and also parameters that affect pollutant emissions such as adiabatic flame temperature and the combustion products. For instance, according to [20] the thermal $\mathrm{NO}_{\mathrm{x}}$ production rate doubles for every $90 \mathrm{~K}$ temperature increase beyond $2100 \mathrm{~K}$.

In this study, a method presented in authors previous work [21] is used for calculation of polynomial coefficients for liquid fuels from the available ideal gas thermodynamic coefficients taken from [22]. Precision of presented liquid phase polynomial coefficients are improved. To show the reliability of the method, a comparison is made with polynomial coefficients which are directly derived from the actual liquid phase thermodynamic properties taken from the thermodynamic tables of NIST obtained from their renown software Refprop [23]. Calculation procedure of the unburned mixture temperature and mixture thermodynamic properties are presented for different fuel/ air ratios and fuel compositions allowing any reactant species to be included in the unburned mixture. The required liquid phase polynomial coefficients for 14 most frequently used chemical compounds such as methanol, ethanol, isooctane, toluene, which represent surrogates of diesel, gasoline jet-A and other frequently used liquid fuels are tabulated in famous NASA format to provide a reference for researchers of combustion and internal 
combustion engines. Effects of equivalence ratio and unburned mixture temperature on enthalpy, specific heat and entropy of unburned fuel-air mixtures as well as effects of phase change on adiabatic flame temperature are presented showing that correct determination of the polynomial coefficients is substantial to obtain thermodynamic properties, flame temperature and accordingly exhaust species accurately which would improve the precision of any thermo-economic and thermo-environmental model.

\section{Theoretical Model}

Equations (1)-(3) give molar specific heat, enthalpy and entropy respectively for each individual species $i$ in the reactant mixture. $\mathrm{a}_{1}-\mathrm{a}_{7}$ are the so called NASA polynomial coefficients of each species which are given in Table 2 .

$$
\begin{aligned}
& \frac{\bar{c}_{p, i}}{\bar{R}_{u}}=a_{1, i}+a_{2, i} T+a_{3, i} T^{2}+a_{4, i} T^{3}+a_{5, i} T^{4}[\mathrm{~kJ} / \mathrm{mol}] \\
& \frac{\overline{\mathrm{h}}_{\mathrm{i}}}{\overline{\mathrm{R}}_{\mathrm{u}} \mathrm{T}}=\mathrm{a}_{1, i}+\frac{\mathrm{a}_{2, \mathrm{i}}}{2} \mathrm{~T}+\frac{\mathrm{a}_{3, \mathrm{i}}}{3} \mathrm{~T}^{2}+\frac{\mathrm{a}_{4, \mathrm{i}}}{4} \mathrm{~T}^{3}+\frac{\mathrm{a}_{5, \mathrm{i}}}{5} \mathrm{~T}^{4}+\frac{\mathrm{a}_{6, \mathrm{i}}}{\mathrm{T}}[\mathrm{kJ} / \mathrm{mol}](2) \\
& \overline{\overline{\mathrm{s}}}_{\mathrm{i}}^{\circ}=\mathrm{a}_{1, i} \text { In } \mathrm{T}+\mathrm{a}_{2, \mathrm{i}} \mathrm{T}+\frac{\mathrm{a}_{3, \mathrm{i}}}{2} \mathrm{~T}^{2}+\frac{\mathrm{a}_{4, \mathrm{i}}}{3} \mathrm{~T}^{3}+\frac{\mathrm{a}_{5, \mathrm{i}}}{4} \mathrm{~T}^{4}+\mathrm{a}_{7, \mathrm{i}}[\mathrm{kJ} / \mathrm{mol}](3) \\
& \mathrm{R}_{\mathrm{u}}
\end{aligned}
$$

where $\overline{\mathrm{R}}_{\mathrm{u}}$ is the molar universal gas constant equal to $8.31434 \mathrm{~kJ} / \mathrm{kmol} \mathrm{K}$ defined as $\bar{R}_{u}=R_{i} M_{i}$ where $M_{i}$ denotes the molecular weight of the unburned reactant. After obtaining the thermodynamic values for each species $i$, thermodynamic properties of unburned fuel-air mixture at a certain temperature can be calculated according to the molar ratios $y_{i}$ as below:
Here, $y_{i}$ stands for the mole fractions of four constituents of air $\left(\mathrm{O}_{2}, \mathrm{~N}_{2}, \mathrm{H}_{2} \mathrm{O}\right.$ and $\mathrm{Ar}$ ) and fractions of constituents of fuel if composed of more than one species. If present, any additional reactant species can be included in Eqs. (4)-(6). When the equivalence ratio is known, fuel and air mass ratios can be obtained as follows:

$$
\phi=\mathrm{FA} / \mathrm{FA}_{\mathrm{s}}
$$

Here, FA is the fuel-air ratio and FA is the stoichiometric fuel-air ratio of the fuel. After obtaining the specific heats of air and fuel separately, temperature of the unburned fuel mixture can be calculated as follows:

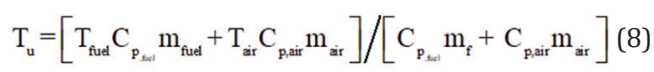

Here, $\mathrm{T}_{\text {fuel }}$ is fuel temperature, $\mathrm{C}_{\mathrm{p} \text {,fuel }}$ is the fuel constant pressure specific heat, $\mathrm{m}_{\text {fuel }}$ is the mass of fuel, $m_{\text {air }}$ is the amount of total combustion air, and $\mathrm{C}_{\mathrm{p} \text {,air }}$ is the constant pressure specific heat corresponding to $\mathrm{T}_{\text {air }}$. If additional species exist, it should be included in Eq. (8) just as fuel and air.

For an arbitrarily selected liquidfuel toluene, thermodynamic properties obtained from Refprop are given in Figure 1. The validation of Refprop data and reference states given to Refprop are done using Gaseq unburned results at dedicated temperature and pressure. The pressure entered in Gaseq is the pressure given by Refprop 0,10577 atm at an arbitrarily selected temperature of $320 \mathrm{~K}$. At this pressure and temperature, Gaseq gives the enthalpy as $568,18 \mathrm{~kJ} / \mathrm{kg}$ matches well with that of NIST Refprop, 567,28 kJ/kg, shown in Figure 1.

$$
\begin{aligned}
& \mathrm{c}_{\mathrm{p}, \mathrm{u}}=\mathrm{m}_{\text {air }} \mathrm{c}_{\mathrm{p}, \text { air }}+\mathrm{m}_{\text {ful }} \mathrm{c}_{\mathrm{p}, \mathrm{fuel}}=\mathrm{m}_{\text {air }}\left(\frac{1}{\mathrm{M}_{\text {air }}} \sum_{\mathrm{i}=1}^{4} \mathrm{y}_{\mathrm{i}} \overline{\mathrm{c}_{\mathrm{p}_{\mathrm{i}}}}\right)+\mathrm{m}_{\text {full }}\left(\sum_{\mathrm{i}=1}^{\mathrm{n}} \frac{\mathrm{y}_{\mathrm{i}} \overline{\mathrm{c}_{\mathrm{p}}}}{\mathrm{M}_{\mathrm{i}}}\right) \quad[\mathrm{kJ} / \mathrm{kg}] \\
& \mathrm{h}_{\mathrm{u}}=\mathrm{m}_{\text {air }} \mathrm{h}_{\text {air }}+\mathrm{m}_{\text {fuel }} \mathrm{h}_{\text {fued }}=\mathrm{m}_{\mathrm{arr}}\left(\frac{1}{\mathrm{M}_{\mathrm{air}}} \sum_{\mathrm{i}=1}^{4} \mathrm{y}_{\mathrm{i}} \overline{\mathrm{h}}_{\mathrm{i}}\right)+\mathrm{m}_{\text {finel }}\left(\sum_{\mathrm{i}=1}^{\mathrm{n}} \frac{\mathrm{y}_{\mathrm{i}} \overline{\mathrm{h}}_{\mathrm{i}}}{\mathrm{M}_{\mathrm{i}}}\right) \quad[\mathrm{kJ} / \mathrm{kg}] \\
& \mathrm{s}_{\mathrm{u}}=\mathrm{m}_{\text {air }} \mathrm{s}_{\text {air }}+\mathrm{m}_{\text {fuel }} \mathrm{s}_{\text {fuel }}=\mathrm{m}_{\text {air }}\left(\frac{1}{\mathrm{M}_{\text {air }}} \sum_{\mathrm{i}=1}^{4} \mathrm{y}_{\mathrm{i}} \mathrm{s}_{\mathrm{i}}^{-\mathrm{o}}\right)+\mathrm{m}_{\text {fuel }}\left(\sum_{\mathrm{i}=1}^{\mathrm{n}} \frac{\stackrel{-0}{\mathrm{y}_{\mathrm{i}}} \mathrm{s}_{\mathrm{i}}^{\mathrm{m}}}{\mathrm{M}_{\mathrm{i}}}\right) \quad[\mathrm{kJ} / \mathrm{kg}]
\end{aligned}
$$


2: toluene: Saturation points (at equilibrium)

\begin{tabular}{|c|c|c|c|c|c|c|c|c|c|c|}
\hline & $\begin{array}{c}\text { Temperature } \\
\text { (K) }\end{array}$ & $\begin{array}{l}\text { Pressure } \\
\text { [atm] }\end{array}$ & $\begin{array}{l}\text { Liquid } \\
\text { Enthalpy } \\
(\mathrm{kJ} / \mathrm{kg})\end{array}$ & $\begin{array}{l}\text { Vapor } \\
\text { Enthalpy } \\
(\mathrm{kJ} / \mathrm{kg})\end{array}$ & $\begin{array}{c}\text { Liquid } \\
\text { Entropy } \\
(\mathrm{kJ} / \mathrm{kg}-\mathrm{K})\end{array}$ & $\begin{array}{c}\text { Vapor } \\
\text { Entropy } \\
(\mathrm{kJ} / \mathrm{kg}-\mathrm{K})\end{array}$ & $\begin{array}{c}\text { Liquid } \\
C_{p} \\
(\mathrm{kN} / \mathrm{kg} \cdot \mathrm{K})\end{array}$ & $\begin{array}{c}\text { Vapor } \\
C_{p} \\
{[\mathrm{~kJ} / \mathrm{kg} \cdot \mathrm{K})}\end{array}$ & $\begin{array}{c}\text { Heat of Vapor. } \\
{[(\mathrm{kJ} / \mathrm{kg})}\end{array}$ & Molar Mass \\
\hline 1 & 273,15 & 0,0089127 & 87,331 & 515,47 & 0,98853 & 2,5560 & 1,6254 & 1,0286 & 428,14 & 92,138 \\
\hline 2 & 290,00 & 0,024213 & 115,13 & 533,21 & 1,0873 & 2,5289 & 1,6751 & 1,0975 & 418,08 & 92,138 \\
\hline 3 & 320,00 & 0,10577 & 166,82 & 567,28 & 1,2568 & 2,5082 & 1,7720 & 1,2219 & 400,46 & 92,138 \\
\hline 4 & 340,00 & 0,23850 & 202,96 & 591,61 & 1,3663 & 2,5094 & 1,8413 & 1,3065 & 388,65 & 92,138 \\
\hline 5 & 360,00 & 0,48362 & 240,52 & 617,10 & 1,4735 & 2,5196 & 1,9136 & 1,3925 & 376,58 & 92,138 \\
\hline 6 & 380,00 & 0,89896 & 279,57 & 643,60 & 1,5789 & 2,5369 & 1,9886 & 1,4803 & 364,04 & 92,138 \\
\hline 7 & 383,40 & 0,99140 & 286,36 & 648,20 & 1,5966 & 2,5404 & 2,0016 & 1,4955 & 361,84 & 92,138 \\
\hline
\end{tabular}

Figure 1. Output of REFPROP for Thermodynamic Properties of Toluene at Dedicated Temperatures and Reference States

However, entropy of Refprop, 2,5082 $\mathrm{kJ} / \mathrm{kg} \mathrm{K}$, verified from Goodwin [24] seems inconsistent that of Gaseq, 3,75706 kJ/kg $\mathrm{K}$. This is because entropy of Gaseq given at $320 \mathrm{~K}$ and $0,10577 \mathrm{~atm}$ is for ideal gas phase and therefore needs the following correction given by Eq.(9) according to the equation of Gibbs for liquid state entropy at equilibrium state and the heat of vaporization at corresponding temperature:
Many handbooks set the arbitrary state point so that the values of these properties are positive for most liquid or gas states. The properties of enthalpy and entropy are computed as differences relative to some arbitrary reference state [26]. In this study, the liquid phase reference state enthalpies and entropies given to Refprop are set exactly to their values at $298,15 \mathrm{~K}$ and 1 bar which are calculated by substituting gas phase enthalpy and entropy values

$$
\begin{array}{ll}
\mathrm{s}_{\text {liq }}=\mathrm{s}_{\text {gas } @ 0,10577 \text { atm }}-\Delta \mathrm{h}_{\text {vap }} / \mathrm{T}=3,75706-400,46 / 320=2,506 & {[\mathrm{~kJ} / \mathrm{kgK}]} \\
\mathrm{h}_{\text {liq }}=\mathrm{h}_{\text {gas }}-\Delta \mathrm{h}_{\text {vap }}=568,18-400,46=167,72 & {[\mathrm{~kJ} / \mathrm{kg}]}
\end{array}
$$

Heat of vaporization in Eq. (9) and Eq. (10) can be calculated with Eq. (11) using the coefficients $\mathrm{C}_{1}-\mathrm{C}_{4}$ and reduced temperature $\mathrm{T}_{\mathrm{r}}=\mathrm{T} / \mathrm{T}_{\mathrm{c}}$. Here, $\mathrm{T}$ is the temperature in Kelvin and $\mathrm{T}_{c}$ is critical temperature taken from [25]. $\mathrm{C}_{3}$ and $\mathrm{C}_{4}$ are equal to zero for the fuels investigated. Other variables used in Eq. (11) are tabulated in Table 1.

$$
\Delta \mathrm{h}_{\text {vap }}=\mathrm{C}_{1}\left(1-\mathrm{T}_{r}\right)^{\mathrm{C}_{2}+\mathrm{C}_{3} \mathrm{~T}_{t}+\mathrm{C}_{t} \mathrm{~T}_{t}^{2}}
$$

In thermodynamics, the absolute values of enthalpy, entropy, and energy at a single state point are meaningless. It is only the difference between two different state points matters. Thus, the value for a single state point can be set to any arbitrary value. at 298,15 K and 1 bar into Eqs. (9)-(10). Gas phase enthalpy and entropy values at 298,15 K and 1 bar are obtained with Gaseq software using gas phase thermodynamic coefficients $\mathrm{a}_{1}-\mathrm{a}_{7}$ for $\mathrm{T}=300-100 \mathrm{~K}$ given in Table 2. Calculated liquid phase reference enthalpy and entropy values, which are used to obtain properties from Refprop relative to NASA reference states, are presented in Table 1.

Comparison of Table 3 and Table 4 show that enthalpy and entropy values calculated by Refprop according to the reference enthalpy and entropy data given in Table 1 can safely be used in obtaining liquid phase polynomial coefficients and will successfully represent the thermodynamic 
Table 1. Parameters for Vaporization Enthalpy Equation and Reference Values for Enthalpy and Entropy Obtained from the Calculated Liquid Phase $a_{1}-a_{7}$ Coefficients at 298,15 K and 1 bar

\begin{tabular}{|l|c|c|c|c|c|c|c|c|}
\hline & $\mathbf{C}_{\mathbf{1}}$ & $\mathbf{C}_{\mathbf{2}}$ & $\mathbf{T}_{\mathbf{c}}$ & $\mathbf{h}_{\text {liq }} \mathbf{k J} / \mathbf{k g}$ & $\mathbf{h}_{\text {gas }} \mathbf{k J} / \mathbf{k g}$ & $\begin{array}{c}\mathbf{s}_{\text {liq }} \mathbf{k J /} \\
\mathbf{k g K}\end{array}$ & $\begin{array}{c}\mathbf{S}_{\text {gas }} \mathbf{k J} / \\
\mathbf{k g K}\end{array}$ & $\begin{array}{c}\Delta \mathbf{h}_{\text {vap- }} \\
\text { REFPROP }\end{array}$ \\
\hline Toluene & 49,507 & 0,37742 & 591,75 & 129,84 & 542,69 & 2,0883 & 3,4730 & 412,85 \\
\hline $\mathbf{n}$-Decane & 66,126 & 0,39797 & 617,7 & $-2114,76$ & $-1753,72$ & 2,6249 & 3,8358 & 361,04 \\
\hline Benzene & 45,346 & 0,39053 & 689 & 627,76 & 1061,32 & 1,9950 & 3,4492 & 433,56 \\
\hline $\mathbf{n}$-Pentane & 39,109 & 0,38681 & 469,7 & $-2477,25$ & $-2210,96$ & 3,9075 & 5,1360 & 366,29 \\
\hline Methanol & 50,451 & 0,33594 & 512,5 & $-7445,03$ & $-6276,03$ & 3,5617 & 7,4825 & 1169 \\
\hline Hexane & 44,544 & 0,39002 & 507,6 & $-2302,9$ & $-1937,43$ & 3,2859 & 4,5117 & 365,47 \\
\hline
\end{tabular}

properties in consistency with Gaseq.

Properties of all fuels presented in Table 4 are obtained from the calculated liquid phase polynomial coefficients $a_{1}-a_{7}$. To obtain liquid phase polynomial coefficients, enthalpy values at 6 evenly distributed temperature in the range of $273,15 \mathrm{~K}$ to $\mathrm{T}_{\text {sat }}$ are taken from Table 3 which presents the liquid phase thermodynamic properties calculated with Refprop. These enthalpy values are used to solve the equation: $\mathrm{k} \times \mathrm{A}$ $=\mathrm{B}$ for $\mathrm{k}$ to obtain $\mathrm{a}_{1}-\mathrm{a}_{6}$ where

$$
A=\left[\begin{array}{llllll}
1 & T_{1} / 2 & T_{1}^{2} / 3 & T_{1}^{3} / 4 & T_{1}^{4} / 5 & 1 / T_{1} \\
1 & T_{2} / 2 & T_{2}^{2} / 3 & T_{2}^{3} / 4 & T_{2}^{4} / 5 & 1 / T_{2} \\
1 & T_{3} / 2 & T_{3}^{2} / 3 & T_{3}^{3} / 4 & T_{3}^{4} / 5 & 1 / T_{3} \\
1 & T_{4} / 2 & T_{4}^{2} / 3 & T_{4}^{3} / 4 & T_{4}^{4} / 5 & 1 / T_{4} \\
1 & T_{5} / 2 & T_{5}^{2} / 3 & T_{5}^{3} / 4 & T_{5}^{4} / 5 & 1 / T_{5} \\
1 & T_{6} / 2 & T_{6}^{2} / 3 & T_{6}^{3} / 4 & T_{6}^{4} / 5 & 1 / T_{6}
\end{array}\right]
$$

Here $h_{u}$ is the total enthalpy of reactants, $h_{b}$ is the enthalpy of the products and $C_{p}$ is the specific heat of the burnt gas. Burned gas properties are calculated according to the chemical equilibrium method proposed by [6] and [27] considering 10 product species. Iteratively, adiabatic flame temperature is obtained by:

$$
\mathrm{AFT}^{n+1}=\mathrm{AFT}^{n}+\text { DeltaT }
$$

For each iteration $(n), h_{u^{\prime}} h_{b}$ and $C_{p}$ are reevaluated until DeltaT becomes less than

$$
B=\left[\begin{array}{l}
h_{1} /\left(R T_{1}\right) \\
h_{2} /\left(R T_{1}\right) \\
h_{3} /\left(R T_{1}\right) \\
h_{4} /\left(R T_{1}\right) \\
h_{5} /\left(R T_{1}\right) \\
h_{6} /\left(R T_{1}\right)
\end{array}\right] \quad k=\left[\begin{array}{c}
a_{1} \\
a_{2} \\
a_{3} \\
a_{4} \\
a_{5} \\
a_{6}
\end{array}\right]
$$

and $\mathrm{h}$ in $\mathrm{kJ} / \mathrm{kg}$. To obtain $\mathrm{a}_{7}$, Eq. (8) is used. As there is only one unknown, one temperature value is enough to solve $a_{7}$ from Eq. (13) below:

$\left.a_{7}=-\left[a_{1} \log \left(T_{1}\right)+a_{2} T_{1}+a_{3} T_{1}^{2} / 2+a_{4} T_{1}^{3} / 3+a_{5} T_{1}^{4} / 4\right)-\frac{s_{1}}{R}\right](13)$

where $s_{1}$ is the liquid phase entropy at temperature $\mathrm{T}_{1}$. Using unburned mixture enthalpy, adiabatic flame temperature can be obtained minimizing DeltaT as follows:

$$
\text { DeltaT }=\left(h_{v}-h_{b}\right) / C_{p}
$$

an acceptable tolerance which is taken as $10^{-5}$ in this study.

\section{Results and Discussion}

In order to determine liquid phase polynomial coefficients for toluene, n-decane, benzene, n-pentane and methanol and hexane, the actual enthalpy are entropy values taken from Refprop which are presented in Table 3. These values are obtained using the liquid phase reference states given in Table 1. Ideal gas thermodynamic data for low temperature (300-1000 K) [22] and calculated liquid 
phase data are presented in Table 2 . To validate the obtained liquid phase thermodynamic data, thermodynamic properties are calculated using Eqs. (1)-(3) using the obtained liquid phase data given in Table 2. The values are presented in Table 4. Refprop values given in Table 3 and calculated values given in Table 4 are very close with an average absolute deviation in enthalpy $|0.0011 \%|$, entropy $|0.0061 \%|$

Table 2. Calculated Liquid Phase Coefficients $a_{1}$ - $a_{7}$ from Results of REFPROP for 273,15 $\mathrm{K}$ to the Saturation Temperature at 1 bar and Gas Phase Coefficients for 300 to $1000 \mathrm{~K}$

\begin{tabular}{|c|c|c|c|c|c|c|c|c|}
\hline Fuels & $\mathrm{T}(\mathrm{K})$ & $a_{1}$ & $a_{2}$ & $a_{3}$ & $a_{4}$ & $a_{5}$ & $a_{6}$ & $a_{7}$ \\
\hline \multirow{2}{*}{$\mathrm{CH}_{3} \mathrm{OH}$} & $273-\mathrm{T}_{\text {sat }}$ & $-646,7031$ & 8,515793 & $-0,04147624$ & 8,96586E-05 & $-7,23619 \mathrm{E}-08$ & 9022,0516 & 2353,7397 \\
\hline & $300-1000$ & 2,660115 & 0,007342 & $7,17005 \mathrm{E}-06$ & $-8,79319 \mathrm{E}-09$ & $2,39057 \mathrm{E}-12$ & $-25353,48$ & 11,23263 \\
\hline \multirow{2}{*}{$\mathrm{C}_{2} \mathrm{H}_{5} \mathrm{OH}$} & $273-\mathrm{T}_{\text {sat }}$ & $-699,9972$ & 9,176359 & $-0,04476726$ & $9,76705 \mathrm{E}-05$ & $-7,98241 \mathrm{E}-08$ & 7602,1751 & 2553,734835 \\
\hline & $300-1000$ & 0,1791061 & 0,030906 & $-1,936 \mathrm{E}-05$ & 6,31831E-09 & $-8,53167 \mathrm{E}-13$ & $-29567,061$ & 24,47159 \\
\hline \multirow{2}{*}{$\mathrm{C}_{5} \mathrm{H}_{12}$} & $273-\mathrm{T}_{\text {sat }}$ & 156639 & $-2165,53$ & 11,2226243 & $-0,025836427$ & $2,22945 \mathrm{E}-05$ & -9084275 & -561380 \\
\hline & $300-1000$ & 10,04976 & $-0,019352$ & $1,4755 \mathrm{E}-04$ & $-1,69026 \mathrm{E}-07$ & 6,289703E-11 & $-21454,04$ & $-12,12254$ \\
\hline \multirow{2}{*}{$\mathrm{C}_{6} \mathrm{H}_{6}$} & $283-\mathrm{T}_{\text {sat }}$ & 391,2617 & $-4,75421$ & 0,02240288 & $-4,67099 \mathrm{E}-05$ & $3,65755 \mathrm{E}-08$ & $-22325,22$ & $-1448,37$ \\
\hline & $300-1000$ & $-3,13801$ & 0,04723 & $-0,0296 \mathrm{E}-04$ & $-0,03263 \mathrm{E}-06$ & $1,71869 \mathrm{E}-11$ & 8890,031 & 36,57573 \\
\hline \multirow{2}{*}{$\mathrm{C}_{6} \mathrm{H}_{14}$} & $273-\mathrm{T}_{\text {sat }}$ & $-3959,3863$ & 51,551371 & $-0,250134802$ & 0,000538815 & $-4,34379 \mathrm{E}-07$ & 215399,833 & 14438,617 \\
\hline & $300-1000$ & 1,836174 & 0,0509846 & $1,2596 \mathrm{E}-05$ & $-4,42836 \mathrm{E}-08$ & $1,872237 \mathrm{E}-11$ & $-22927,49$ & 20,88145 \\
\hline \multirow{2}{*}{$\mathrm{C}_{7} \mathrm{H}_{8}$} & $273-\mathrm{T}_{\text {sat }}$ & 13,04711 & 0,02703 & $-0,00016714$ & 6,89549E-07 & $-7,17719 \mathrm{E}-10$ & $-3199,95$ & $-56,5045$ \\
\hline & $300-1000$ & $-2,53688$ & 0,052899 & 1,4039E-06 & $-4,07623 \mathrm{E}-08$ & 2,037752E-11 & 4477,848 & 37,41512 \\
\hline \multirow{2}{*}{$\mathrm{C}_{7} \mathrm{H}_{16}$} & $273-\mathrm{T}_{\text {sat }}$ & 62,87312 & 0,00066 & $-0,00202554$ & 8,56744E-06 & $-9,62659 \mathrm{E}-09$ & $-44656,880$ & $-303,351$ \\
\hline & $300-1000$ & 11,1533 & $-0,009494$ & $1,9557 \mathrm{E}-04$ & $-2,49754 \mathrm{E}-07$ & $9,848777 \mathrm{E}-11$ & $-26768,89$ & $-15,90968$ \\
\hline \multirow{2}{*}{$\mathrm{C}_{8} \mathrm{H}_{18}$} & $273-\mathrm{T}_{\text {sat }}$ & 138,4995 & $-1,39505$ & 0,006389919 & $-1,26202 \mathrm{E}-05$ & 9,42853E-09 & $-45321,297$ & $-530,156$ \\
\hline & $300-1000$ & 1,1759 & 0,073227 & 1,1217E-05 & $-5,564996 \mathrm{E}-08$ & 2,463636E-11 & $-28667,47$ & 22,36527 \\
\hline \multirow{2}{*}{$\mathrm{C}_{9} \mathrm{H}_{12}$} & $273-\mathrm{T}_{\text {sat }}$ & 10,59797 & $-2,64 \mathrm{E}-06$ & 0,000262034 & $-4,98291 \mathrm{E}-07$ & $3,98255 \mathrm{E}-10$ & $-9294,742$ & $-38,485$ \\
\hline & $300-1000$ & 4,674885 & 0,019894 & $1,25511-04$ & $-1,817844 \mathrm{E}-07$ & 7,472863E-11 & $-2122,436$ & 11,830198 \\
\hline \multirow{2}{*}{$\mathrm{C}_{10} \mathrm{H}_{8}$} & $273-\mathrm{T}_{\text {sat }}$ & 8,819087 & $1,36 \mathrm{E}-05$ & 0,000280991 & $-5,16720 \mathrm{E}-07$ & $3,43067 \mathrm{E}-10$ & 7122,5606 & $-40,2164$ \\
\hline & $300-1000$ & $-1,049195$ & 0,046297 & 7,07592E-05 & $-1,38408 \mathrm{E}-07$ & $6,204754 \mathrm{E}-11$ & 15984,9 & 30,21216 \\
\hline \multirow{2}{*}{$\mathrm{C}_{10} \mathrm{H}_{14}$} & $273-\mathrm{T}_{\text {sat }}$ & 9,904477 & $-8,87 \mathrm{E}-06$ & 0,000286849 & $-4,93461 \mathrm{E}-07$ & 3,56033E-10 & 2333,7534 & $-37,1121$ \\
\hline & $300-1000$ & 5,029490 & 0,006937 & $2,0424 \mathrm{E}-04$ & $-2,79971 \mathrm{E}-07$ & 1,138555E-10 & 9970,859 & 10,68859 \\
\hline \multirow{2}{*}{$\mathrm{C}_{10} \mathrm{H}_{22}$} & $273-T_{\text {sat }}$ & 7182,861 & $-93,4367$ & 0,456722542 & $-0,00098965$ & $8,02635 \mathrm{E}-07$ & $-482873,58$ & $-26164,3$ \\
\hline & $300-1000$ & 15,43282 & $-0,013298$ & 2,82481E-04 & $-3,65923 \mathrm{E}-07$ & $1,45372 \mathrm{E}-10$ & $-35863,28$ & $-27,94543$ \\
\hline \multirow{2}{*}{$\mathrm{C}_{11} \mathrm{H}_{10}$} & $273-\mathrm{T}_{\text {sat }}$ & 9,422619 & $1,14 \mathrm{E}-05$ & 0,00028288876 & $-4,85268 \mathrm{E}-07$ & $3,00461 \mathrm{E}-10$ & 17030,125 & $-37,9392$ \\
\hline & $300-1000$ & 1,591975 & 0,034402 & $1,24038 \mathrm{E}-04$ & $-2,01416 \mathrm{E}-07$ & $8,67316 \mathrm{E}-11$ & 25890,536 & 23,75374 \\
\hline \multirow{2}{*}{$\mathrm{C}_{16} \mathrm{H}_{34}$} & $273-\mathrm{T}_{\text {sat }}$ & 23,7035 & 8,87E-05 & 0,000653645 & $-1,28346 \mathrm{E}-06$ & $8,86626 \mathrm{E}-10$ & $-65348,85$ & $-90,7578$ \\
\hline & $300-1000$ & $-2,281474$ & 0,185128 & $-9,9178 \mathrm{E}-05$ & 1,433984E-08 & $3,732305 \mathrm{E}-12$ & $-51744,93$ & 56,00249 \\
\hline $\mathrm{N}_{2}$ & $300-1000$ & 3,298677 & 0,001408 & $-0,0396 \mathrm{E}-04$ & $0,056415 \mathrm{E}-07$ & $-0,02445 \mathrm{E}-10$ & $-1020,900$ & 3,950372 \\
\hline $\mathrm{O}_{2}$ & $300-1000$ & 3,212936 & 0,001128 & $-5,7562 \mathrm{E}-07$ & 1,313877E-09 & $-8,76855 \mathrm{E}-13$ & $-1005,249$ & 6,034737 \\
\hline $\mathrm{H}_{2} \mathrm{O}$ & $300-1000$ & 3,386842 & 0,003475 & $-6,3547 \mathrm{E}-06$ & 6,96858E-09 & $-2,50659 \mathrm{E}-12$ & $-30208,11$ & 2,590232 \\
\hline $\mathrm{Ar}$ & $300-1000$ & 2,5 & 0 & 0 & 0 & 0 & $-745,375$ & 4,366 \\
\hline
\end{tabular}


and specific heat $|0.3918 \%|$. The absolute average deviation is $|0.133 \%|$. Further, comparison of Table 3 and Table 4 shows that liquid and ideal gas thermodynamic coefficients are well interchangeable temperature limit) and ended with the saturation temperature of each fuel at $1 \mathrm{bar}$.

Figure 2 shows the variation of enthalpy of unburned fuel-air mixture with equivalence ratios changing between

Table 3. NIST Refprop Liquid Phase Thermodynamic Data for Toluene, n-Decane, Benzene, $n$-Pentane, Methanol and Hexane

\begin{tabular}{|c|c|c|c|c|c|c|c|}
\hline \multicolumn{4}{|c|}{ Toluene } & \multicolumn{4}{|c|}{ n-Decane } \\
\hline $\mathbf{T}[\mathrm{K}]$ & $C_{p} \mathrm{~kJ} / \mathrm{kgK}$ & h $\mathrm{kJ} / \mathrm{kg}$ & s $\mathrm{kJ} / \mathrm{kgK}$ & $\mathbf{T}[\mathrm{K}]$ & $C_{p} \mathrm{~kJ} / \mathrm{kgK}$ & h $\mathrm{kJ} / \mathrm{kg}$ & s $\mathrm{kJ} / \mathrm{kgK}$ \\
\hline 273,15 & 1,6257 & 88,269 & 1,9423 & 273,15 & 2,1040 & $-2168,5$ & 2,4369 \\
\hline 290 & 1,6757 & 116,08 & 2,0410 & 290 & 2,1628 & $-2132,6$ & 2,5645 \\
\hline 320 & 1,7731 & 167,79 & 2,2106 & 305 & 2,2183 & $-2099,7$ & 2,6750 \\
\hline 340 & 1,8425 & 203,95 & 2,3202 & 320 & 2,2760 & $-2065,9$ & 2,7829 \\
\hline 360 & 1,9145 & 241,54 & 2,4275 & 335 & 2,3356 & $-2031,4$ & 2,8885 \\
\hline 380 & 1,9889 & 280,60 & 2,5329 & 345 & 2,3762 & $-2007,8$ & 2,9578 \\
\hline 383,4 & 2,0018 & 287,39 & 2,5506 & 347,3 & 2,3856 & $-2002,3$ & 2,9736 \\
\hline \multicolumn{4}{|c|}{ Benzene } & \multicolumn{4}{|c|}{ n-Pentane } \\
\hline $\mathbf{T}[\mathbf{K}]$ & $C_{p} \mathrm{~kJ} / \mathrm{kgK}$ & h $\mathrm{kJ} / \mathrm{kg}$ & s $\mathrm{kJ} / \mathrm{kgK}$ & $\mathbf{T}[\mathrm{K}]$ & $C_{p} \mathrm{~kJ} / \mathrm{kgK}$ & h $\mathrm{kJ} / \mathrm{kg}$ & s kJ/kgK \\
\hline 283,15 & 1,698 & 602,01 & 1,9064 & 273,15 & 2,2099 & $-2503,8$ & 3,7097 \\
\hline 290 & 1,7144 & 613,70 & 1,9472 & 280 & 2,2371 & $-2488,6$ & 3,7647 \\
\hline 305 & 1,754 & 639,71 & 2,0346 & 290 & 2,2791 & $-2466,0$ & 3,8439 \\
\hline 320 & 1,7977 & 666,36 & 2,1198 & 295 & 2,3011 & $-2454,5$ & 3,8830 \\
\hline 335 & 1,8446 & 693,69 & 2,2032 & 300 & 2,3239 & $-2443,0$ & 3,9218 \\
\hline 350 & 1,8941 & 721,75 & 2,2850 & 305 & 2,3473 & $-2431,3$ & 3,9604 \\
\hline 353,1 & 1,9046 & 727,64 & 2,3017 & 308,2 & 2,3626 & $-2423,7$ & 3,9850 \\
\hline \multicolumn{4}{|c|}{ Methanol } & \multicolumn{4}{|c|}{ Hexane } \\
\hline $\mathrm{T}$ [K] & $\mathrm{C}_{\mathrm{p}} \mathrm{kJ} / \mathrm{kgK}$ & h kJ/kg & s $\mathrm{kJ} / \mathrm{kgK}$ & $\mathbf{T}[\mathrm{K}]$ & $\mathrm{C}_{\mathrm{p}} \mathrm{kJ} / \mathrm{kgK}$ & h $\mathrm{kJ} / \mathrm{kg}$ & s $\mathrm{kJ} / \mathrm{kgK}$ \\
\hline 273,15 & 2,4011 & $-7506,7$ & 3,3460 & 273,15 & 2,1525 & $-2357,9$ & 3,0932 \\
\hline 285 & 2,4593 & $-7477,9$ & 3,4491 & 285 & 2,1982 & $-2332,2$ & 3,1856 \\
\hline 300 & 2,5461 & $-7440,3$ & 3,5774 & 300 & 2,2598 & $-2298,7$ & 3,2999 \\
\hline 310 & 2,6120 & $-7414,5$ & 3,6619 & 310 & 2,3032 & $-2275,9$ & 3,3746 \\
\hline 325 & 2,7223 & $-7374,5$ & 3,7878 & 320 & 2,3482 & $-2252,6$ & 3,4484 \\
\hline 335 & 2,8034 & $-7346,9$ & 3,8715 & 330 & 2,3949 & $-2228,9$ & 3,5213 \\
\hline 336,8 & 2,8186 & $-7341,8$ & 3,8866 & 340,8 & 2,4472 & $-2202,7$ & 3,5992 \\
\hline
\end{tabular}

as one can be converted to the other if vaporization enthalpies are known at each temperature step at which the polynomials are interpolated.

Temperatures in Table 3 and Table 4 are started from $0^{\circ} \mathrm{C}$ (except for benzene because of the Refprop's minimum
0.3 and 1.4 and for unburned mixture temperatures of 300, 500, 700 and 900 Kelvin. According to Figure 2, enthalpy increases almost linearly with unburned mixture temperature. Among the 10 fuels investigated, methanol mixture shows the highest enthalpy change at all temperatures 
Table 4. Calculated with the Calculated $a_{1}-a_{7}$ Data

\begin{tabular}{|c|c|c|c|c|c|c|c|}
\hline \multicolumn{4}{|c|}{ Toluene } & \multicolumn{4}{|c|}{ n-Decane } \\
\hline $\mathrm{T}[\mathrm{K}]$ & $\mathrm{C}_{\mathrm{p}} \mathrm{kJ} / \mathrm{kgK}$ & h kJ/kg & s kJ/kgK & $\mathrm{T}[\mathrm{K}]$ & $\mathrm{C}_{\mathrm{p}} \mathrm{kJ} / \mathrm{kgK}$ & h kJ/kg & s kJ/kgK \\
\hline 273,15 & 1,6259 & 88,270 & 1,9423 & 273,15 & 2,1166 & $-2168,5$ & 2,4369 \\
\hline 290 & 1,6758 & 116,081 & 2,0411 & 290 & 2,1591 & $-2132,6$ & 2,5644 \\
\hline 320 & 1,7736 & 167,792 & 2,2107 & 305 & 2,2264 & $-2099,7$ & 2,6750 \\
\hline 340 & 1,8433 & 203,952 & 2,3202 & 320 & 2,2771 & $-2065,9$ & 2,7832 \\
\hline 360 & 1,9161 & 241,542 & 2,4277 & 335 & 2,3272 & $-2031,4$ & 2,8886 \\
\hline 380 & 1,9902 & 280,605 & 2,5332 & 345 & 2,3941 & $-2007,8$ & 2,9579 \\
\hline 383,4 & 2,0029 & 287,393 & 2,5510 & 347,3 & 2,4174 & $-2002,3$ & 2,4369 \\
\hline \multicolumn{4}{|c|}{ Benzene } & \multicolumn{4}{|c|}{ n-Pentane } \\
\hline $\mathrm{T}[\mathrm{K}]$ & $C_{p} \mathrm{~kJ} / \mathrm{kgK}$ & h $\mathrm{kJ} / \mathrm{kg}$ & s kJ/kgK & $\mathbf{T}[\mathrm{K}]$ & $C_{p} \mathrm{~kJ} / \mathrm{kgK}$ & h kJ/kg & s kJ/kgK \\
\hline 283,15 & 1,6992 & 602,020 & 1,9064 & 273,15 & 2,2519 & $-2503,84$ & 3,7098 \\
\hline 290 & 1,7145 & 613,710 & 1,9472 & 280 & 2,2189 & $-2488,64$ & 3,7647 \\
\hline 305 & 1,7547 & 639,721 & 2,0347 & 290 & 2,2950 & $-2466,04$ & 3,844 \\
\hline 320 & 1,7991 & 666,371 & 2,1200 & 295 & 2,3012 & $-2454,54$ & 3,8833 \\
\hline 335 & 1,8454 & 693,702 & 2,2034 & 300 & 2,3018 & $-2443,04$ & 3,922 \\
\hline 350 & 1,8964 & 721,756 & 2,2853 & 305 & 2,3569 & $-2431,43$ & 3,9604 \\
\hline 353,1 & 1,9080 & 727,652 & 2,3021 & 308,2 & 2,4647 & $-2423,74$ & 3,9855 \\
\hline \multicolumn{4}{|c|}{ Methanol } & \multicolumn{4}{|c|}{ Hexane } \\
\hline $\mathbf{T}[\mathrm{K}]$ & $\mathrm{C}_{\mathrm{p}} \mathrm{kJ} / \mathrm{kgK}$ & h kJ/kg & s kJ/kgK & $\mathbf{T}[\mathrm{K}]$ & $\mathrm{C}_{\mathrm{p}} \mathrm{kJ} / \mathrm{kgK}$ & h kJ/kg & s kJ/kgK \\
\hline 273,15 & 2,3928 & $-7506,747$ & 3,3459 & 273,15 & 2,1249 & $-2357,92$ & 3,0934 \\
\hline 285 & 2,4652 & $-7477,947$ & 3,4491 & 285 & 2,2035 & $-2332,22$ & 3,1854 \\
\hline 300 & 2,5491 & $-7440,346$ & 3,5776 & 300 & 2,2608 & $-2298,72$ & 3,3000 \\
\hline 310 & 2,6124 & $-7414,546$ & 3,6622 & 310 & 2,3006 & $-2275,92$ & 3,3747 \\
\hline 325 & 2,7239 & $-7374,546$ & 3,7882 & 320 & 2,3493 & $-2252,68$ & 3,4485 \\
\hline 335 & 2,8041 & $-7346,906$ & 3,8720 & 330 & 2,4019 & $-2228,92$ & 3,5216 \\
\hline 336,8 & 2,8183 & $-7341,846$ & 3,8870 & 340,8 & 2,4456 & $-2202,72$ & 3,5998 \\
\hline
\end{tabular}

followed by syngas, ethanol, dimethyl ether (dme) and methane mixtures. For $\mathrm{T}_{\mathrm{u}}=300$ $\mathrm{K}$, enthalpy of methanol decreases by $303 \%$ when equivalence ratio is increased from 0.3 to 1.4 . For fuel-air mixtures with surrogates of diesel, gasoline, jet-A and also for isooctane and propane, effect of equivalence ratio on the mixture enthalpy is negligible. For example at $900 \mathrm{~K}$ enthalpy of gasoline is $647 \mathrm{~kJ} / \mathrm{kg}$ at $\Phi=0.3$ and 672.4 $\mathrm{kJ} / \mathrm{kg}$ at $\Phi=1.4$. However, the net effect of temperature change on the enthalpies of diesel, gasoline, jet-A and propane are almost the same.

Figure 3 shows the variation of entropy of unburned fuel-air mixture with equivalence ratios changing between 0.3 and 1.4 and for unburned mixture temperatures of 300, 500, 700 and 900 Kelvin. According to Figure 3, entropy increases almost linearly with unburned mixture temperature as for enthalpy. It can be seen from the figure that, for the same fuel, variation of entropy with equivalence ratio is almost parallel to each other. Among the 10 fuels investigated, syngas-air mixture shows the highest 
Table 5. Liquid Fuel Surrogates Representing Jet-A, Diesel and Gasoline

\begin{tabular}{|c|c|c|c|c|c|c|}
\hline & & & & Surrogate & & Syngas \\
\hline & & & Jet A [29] & Diesel [30] & Gasoline [31] & [28] \\
\hline Carbon monoxide & $\mathrm{CO}$ & & & & & 0,5 \\
\hline Hydrogen & $\mathrm{H}_{2}$ & & & & & 0,5 \\
\hline Isooctane & $\mathrm{C}_{8} \mathrm{H}_{18}$ & & 0,33 & & 0,34 & \\
\hline Toluene & $\mathrm{C}_{7} \mathrm{H}_{8}$ & & 0,243 & & & \\
\hline n-Pentane & $\mathrm{C}_{5} \mathrm{H}_{12}$ & mixture & & & 0,10 & \\
\hline Benzene & $\mathrm{C}_{6} \mathrm{H}_{6}$ & & & & 0,56 & \\
\hline$\alpha$-methylnaphtalene(MNP) & $\mathrm{C}_{11} \mathrm{H}_{10}$ & & & 0,30 & & \\
\hline n-Decane & $\mathrm{C}_{10} \mathrm{H}_{22}$ & & 0,427 & 0,70 & & \\
\hline
\end{tabular}

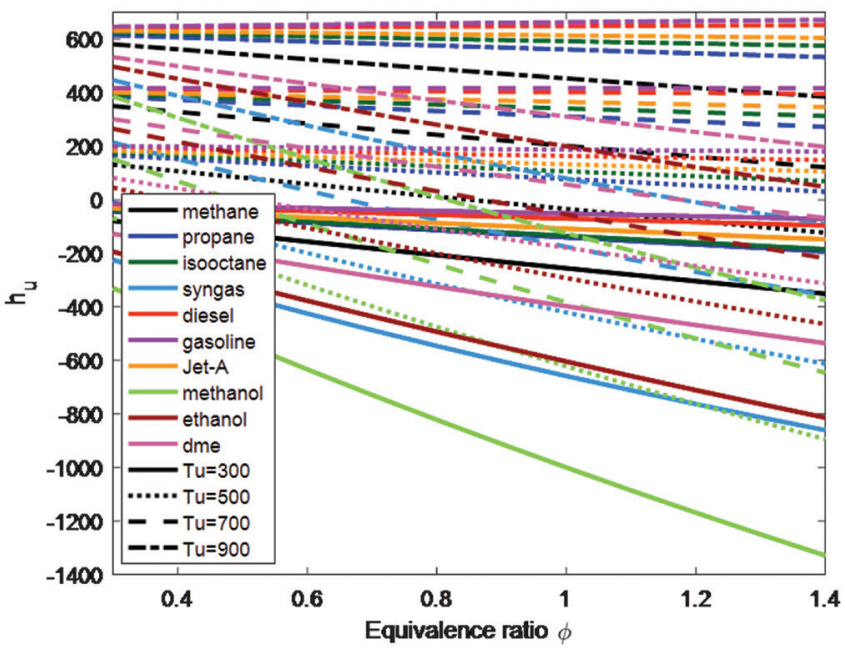

Figure 2. Enthalpy of Unburned Fuel-Air Mixture for Various Fuels at Different Equivalence Ratios and for Different Unburned Mixture Temperatures

entropy change with equivalence ratio at all temperatures followed by methane and methanol mixtures. For unburned mixture temperature of $300 \mathrm{~K}$, entropy increases by $12.2 \%$ for syngas and by $5 \%$ for methane when equivalence ratio is increased from 0.3 to 1.4 . For these mixtures, entropy increases with equivalence ratio. For, diesel, gasoline, jet-A, propane, ethanol, isooctane and dme mixtures, entropy values are very close to each other and slightly decrease with equivalence ratio.

Figure 4 shows the variation of specific heat of unburned fuel-air mixture with equivalence ratios changing between 0.3 and 1.4 and for unburned mixture temperatures of $300,500,700$ and 900 Kelvin. According to Figure 4, specific heat increases linearly with equivalence ratio for each fuel-air mixture. It can be seen from the figure that, for the same fuel, variation of specific heats with equivalence ratio are parallel to each other for unburned temperatures of $300 \mathrm{~K}, 500 \mathrm{~K}, 700 \mathrm{~K}$ and $900 \mathrm{~K}$ as it was in Figure 2 and Figure 3 except for the case that the fuels are methanol, ethanol at $300 \mathrm{~K}$ where both fuels are in their liquid phase. Highest specific 
heat values are for methanol, ethanol, dme, methane and isooctane respectively for gas phase whereas in liquid phase specific heat is highest for methanol, syngas and ethanol which has a significantly higher specific heat than its successors isooctane, methane, jet-A and diesel. According to Figure 4, lowest specific heats are for propane and dme at $300 \mathrm{~K}$.
Figure 5 shows the change of adiabatic flame temperatures of unburned fuel-air mixture for varying equivalence ratios at $300 \mathrm{~K}$ unburned mixture temperature. Unburned properties are calculated with gaseous polynomial coefficients and with calculated liquid fuel coefficients adiabatic flame temperatures are presented comparatively. This is a substantial

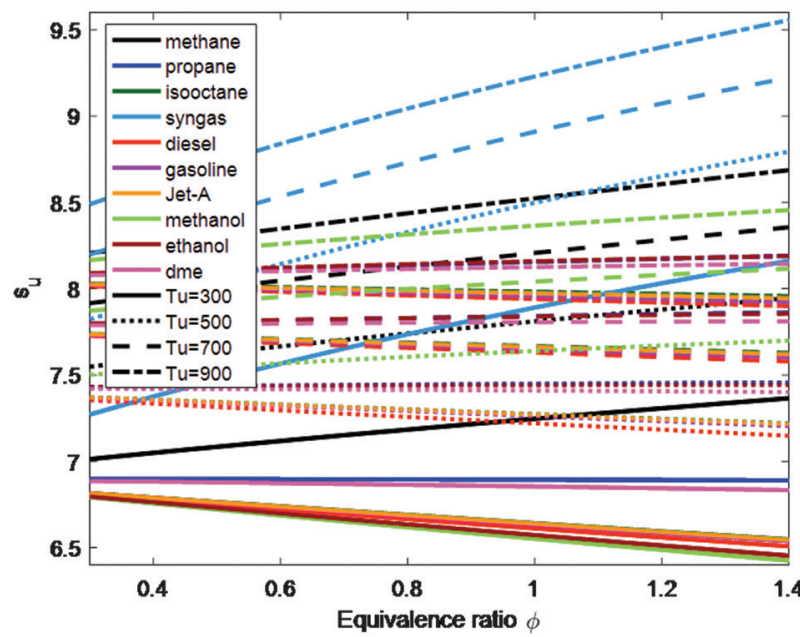

Figure 3. Entropy of Unburned Fuel-Air Mixture for Various Fuels at Different Equivalence Ratios and for Different Unburned Mixture Temperatures

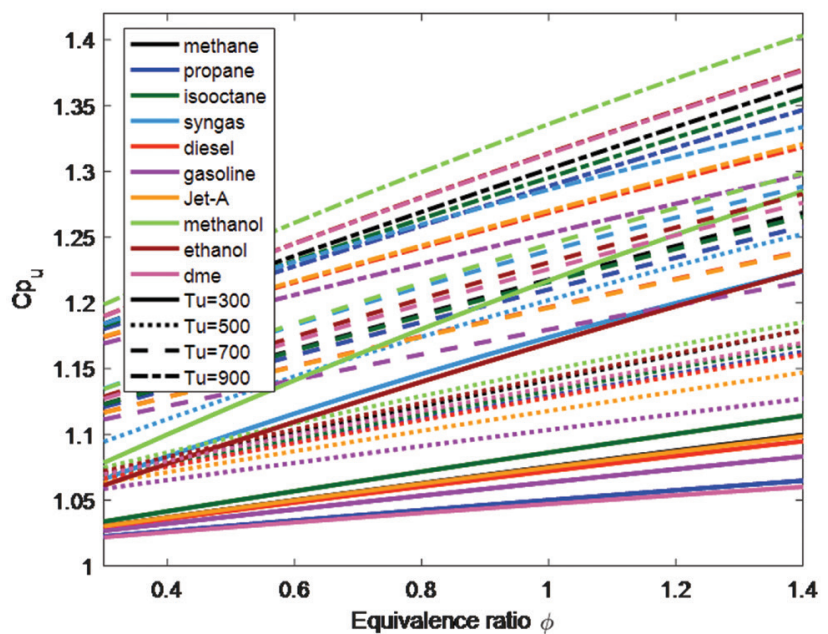

Figure 4. Specific Heat of Unburned Fuel-Air Mixture for Various Fuels at Different Equivalence Ratios and for Different Unburned Mixture Temperatures 
difference in adiabatic flame temperature especially for ethanol and methanol in their gaseous and liquid phases as it was already stated that a $90 \mathrm{~K}$ increase in flame temperature would double the formation of thermal $\mathrm{NO}_{\mathrm{x}}$. At stoichiometry, differences are $41 \mathrm{~K}$ and $70 \mathrm{~K}$ respectively. Using gas phase thermodynamic coefficients would cause important deviations in calculation of performance parameters and pollutant emissions such as $\mathrm{NO}_{\mathrm{x}}$ and $\mathrm{CO}$ which are highly dependent on the flame temperature.

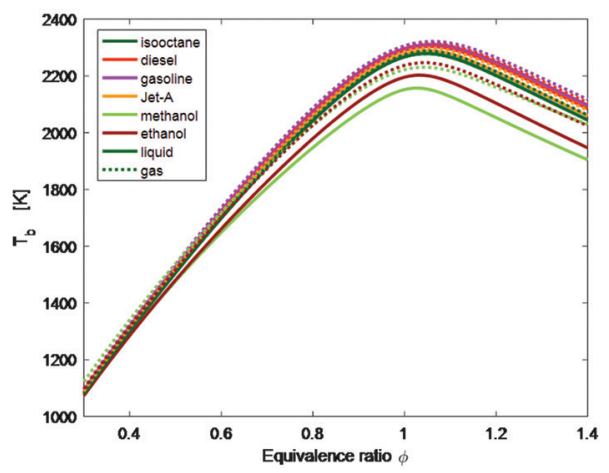

(a) error would cause important deviations in performance estimations and calculation of pollutant emissions such as $\mathrm{NO}_{\mathrm{x}}$ and $\mathrm{CO}$ which are highly dependent on the flame temperature. As a result, to obtain a correct unburned mixture temperature, adiabatic flame temperature and consequently correct performance results and exhaust gas compositions for liquid fuels, liquid phase polynomial data which can be obtained from the available gas polynomial coefficients should be used.

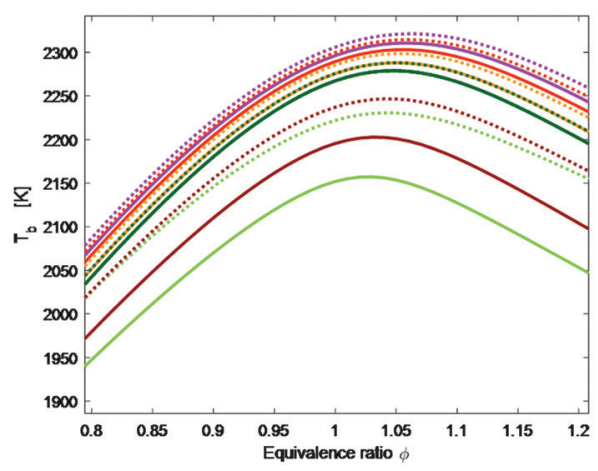

(b)

Figure 5. (a) Adiabatic Flame Temperatures of Unburned Fuel-Air Mixture for Various Fuels at Different Equivalence Ratios for Unburned Mixture Temperature of 300 K. (b) Zoom in Fig. 5(a)

\section{Conclusions}

This paper shows variations of unburned mixture properties with temperature and equivalence ratio for various fuels and states that low temperature ideal gas polynomial coefficients can safely be used for liquid fuels when presented method is applied. Precision of enthalpies and specific heat values of unburned liquid fuels obtained by transformation of the ideal gas polynomial coefficients are satisfactory. For comparison, adiabatic flame temperatures of common liquid fuels are calculated both with available gas phase coefficients and obtained coefficients for liquid phase. According to the results, substantial differences are observed in adiabatic flame temperature especially for ethanol and methanol. Obviously, this

\section{References}

[1] Talay, A. A., Deniz, C. and Durmuşoğlu, Y. (2014). "Gemilerde Verimi Arttırmak İçin Uygulanan Yöntemlerin CO2 Emisyonlarını Azaltmaya Yönelik Etkilerinin Analizi." Journal of ETA Maritime Science 2(1): 61-74.

[2] Doğan, H. E., Arslan, H. and Mehdiyev, R. (2013). "LPG Dönüşümü Yapılmış Bir Dizel Motorunda Azotoksit Oluşumunun Teorik ve Deneysel Olarak İncelenmesi." Journal of ETA Maritime Science 1(2): 27-38.

[3] Öztürk, E. (2013). "Operational Measures For Energy Efficiency In Shipping." Journal of ETA Maritime Science 1(2): 65-72.

[4] Parlak, A. and Kökkülünk, G. (2016). "Bir Gemi Dizel Motorunun Performans 
Ölçümü ve Değerlendirilmesi." Journal of ETA Maritime Science 4(3): 165173.

[5] Ferguson, C. R. (2016). Internal combustion engines : applied thermosciences. New York, John Wiley.

[6] Gordon, S. and McBride, B. J. (1971). Computer program for calculation of complex chemical equilibrium compositions, rocket performance, incident and reflected shocks, and Chapman-Jouguet detonations. Washington,, National Aeronautics and Space Administration.

[7] Gardiner, W. C. (1984). Combustion Chemistry. New York, Springer-Verlag.

[8] Heywood, J. B. (1988). Internal combustion engine fundamentals. New York, US, McGraw-Hill.

[9] Rashidi, M. (1998). "Calculation of equilibrium composition in combustion products." Applied Thermal Engineering 18(3-4): 103109.

[10] Visser, W. P. J. and Kluiters, S. C. A. (1998). Modelling the effects of operating conditions and alternative fuels on gas turbine performance and emissions. Netherlands, NLR National Aerospace Laboratory.

[11] Rakopoulos, C. D., Hountalas, D. T., Tzanos, E. I. and Taklis, G. N. (1994). "A Fast Algorithm for Calculating the Composition of Diesel Combustion Products Using 11 Species ChemicalEquilibrium Scheme." Advances in Engineering Software 19(2): 109-119.

[12] Gonca, G. (2015). "Investigation of the influences of steam injection on the equilibrium combustion products and thermodynamic properties of bio fuels (biodiesels and alcohols)." Fuel 144: 244-258.

[13] Kayadelen, H. K. and Üst, Y. (2017). "Thermodynamic, environmental and economic performance optimization of simple, regenerative, STIG and
RSTIG gas turbine cycles." Energy 121: 751-771.

[14] Kayadelen, H. K. and Üst, Y. (2014). Thermodynamic properties of engine exhaust gas for different kind of fuels. MFMHMT 2014 - The 2014 International Conference on Mechanics, Fluid Mechanics, Heat and Mass Transfer, Interlaken, Switzerland.

[15] Kayadelen, H. K. and Üst, Y. (2014). "Performance and environment as objectives in multi-criterion optimization of steam injected gas turbine cycles." Applied Thermal Engineering (71): 184-196.

[16] Kayadelen, H. K. and Üst, Y. (2013). "Prediction of equilibrium products and thermodynamic properties in H2O injected combustion for CHON type fuels." Fuel 113: 389-401.

[17] Kayadelen, H. K. and Üst, Y. (2014). Thermodynamic properties of engine exhaust gas for different kind of fuels. Computational Problems in Science and Engineering. N. Mastorakis and V. Mladenov. Interlaken, Switzerland, Springer: 247-260.

[18] Reaction Design (2009). CHEMKIN Pro Release 15083. San Diego, CA, Reaction Design.

[19] Morley, C. (2005). "GASEQ, A Chemical Equilibrium Program for Windows Ver. 0.79."

[20] Habib, M. A., Elshafei, M. and Dajani, M. (2008). "Influence of combustion parameters on NOx production in an industrial boiler." Computers \& Fluids (37): 12-23.

[21] Kayadelen, H. K. (2017). Thermodynamic properties of unburned CHON type fuels, fuel mixtures and reactant mixtures. International Conference on Energy and Thermal Engineering, ICTE 2017, İstanbul, Yildiz Technical University.

[22] Kuihua, H. and Chunmei, L. (2007). 
"Kinetic Model and Simulation of Promoted Selective Non-catalytic Reduction by Sodium Carbonate." Chin. J. Chem. Eng. 15(4): 512-519.

[23] Lemmon, E. W., Huber, M. L. and McLinden, M. O. (2013). NIST Standard Reference Database 23: Reference Fluid Thermodynamic and Transport Properties-REFPROP, Version 9.1, Gaithersburg, National Institute of Standards and Technology, Standard Reference Data Program.

[24] Goodwin, R. D. (1989). "Toluene Thermophysical Properties frm 178 $\mathrm{K}$ to $800 \mathrm{~K}$ at pressures to 1000 Bar." J. Phys. Chem Ref. Data 18(4): 15651636.

[25] Poling, B. E. and Thomson, G. H. (2008). Perry's Chemical Engineers Handbook 8th Edition. U.S., McGraw Hill.

[26] Lemmon, E. W., Huber, M. L. and McLinden, M. O. 2013). NIST Reference Fluid Thermodynamic and Transport Properties-REFPROP Version 9.1 User's Guide. Gaithersburg, Maryland, National Institute of Standards and Technology, Standard Reference Data Program.

[27] Olikara, C. and Borman, G. L. (1975). A computer program for calculating properties of equilibrium combustion products with some applications to I.C. engines. Warrendale, Pa., Society of Automotive Engineers.

[28] Lieuwen, T. C., Yang, V. and R. Yetter, Eds. (2010). Synthesis Gas Combustion - Fundamentals and Application. Boca Raton, FL, CRC Press.

[29] Dooley, S., Wona, S. H., Heyne, J., Farouk, T. I., Ju, Y., Dryer, F. L., Kumar, K., Hui, X., Sun, C.-J., Wang, H., Oehlschlaeger, M. A., Iyer, V., Iyer, S., Litzinger, T. A., Santoro, R. J., Malewicki, T. and Brezinsky, K. (2012). "The experimental evaluation of a methodology for surrogate fuel formulation to emulate gas phase combustion kinetic phenomena." Combustion and Flame 159: 14441466.

[30] Pitz, W. J. (2009). Recent Progress in the Development of Diesel Surrogate Fuels. Georgia, U.S., Coordinating Research Council, CRC Inc.

[31] Gauthier, B. M., Davidson, D. F. and Hanson, R. K. (2004). "Shock tube determination of ignition delay times in full-blend and surrogate fuel mixtures." Combustion and Flame 139: 300-311. 